\title{
openheart Relationships among achieved heart rate, $\beta$-blocker dose and long-term outcomes in patients with heart failure with atrial fibrillation
}

\author{
Robert J H Miller, ${ }^{1}$ Jonathan G Howlett, ${ }^{1}$ Michael H Chiu, ${ }^{1}$ Danielle A Southern, ${ }^{2}$ \\ Merril Knudtson, ${ }^{1}$ Stephen B Wilton ${ }^{1,2}$
}

To cite: Miller RJH, Howlett JG, Chiu MH, et al. Relationships among achieved heart rate, $\beta$-blocker dose and long-term outcomes in patients with heart failure with atrial fibrillation. Open Heart 2016:3:e000520. doi:10.1136/openhrt-2016000520

- Additional material is available. To view please visit the journal online (http://dx. doi.org/10.1136/openhrt2016-000520)

Received 15 August 2016 Revised 26 November 2016 Accepted 28 November 2016

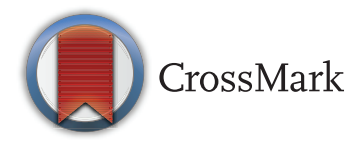

${ }^{1}$ Libin Cardiovascular Institute of Alberta, University of Calgary, Calgary, Alberta, Canada

${ }^{2}$ Department of Community Health Sciences, Calgary Institute for Population and Public Health, University of Calgary, Calgary, Alberta, Canada

Correspondence to Dr Robert JH Miller: robert.miller@ albertahealthservices.ca

\section{ABSTRACT}

Objective: Higher $\beta$-blocker dose and lower heart rate are associated with decreased mortality in patients with systolic heart failure (HF) and sinus rhythm. However, in the $30 \%$ of patients with $\mathrm{HF}$ with atrial fibrillation (AF), whether $\beta$-blocker dose or heart rate predict mortality is less clear. We assessed the association between $\beta$-blocker dose, heart rate and all-cause mortality in patients with $\mathrm{HF}$ and $\mathrm{AF}$.

Methods: We performed a retrospective cohort study in 935 patients $(60 \%$ men, mean age $74,44.7 \%$ with reduced left ventricular ejection fraction (LVEF)) discharged with concurrent diagnoses of $\mathrm{HF}$ and $\mathrm{AF}$. We used Cox models to test independent associations between higher versus lower predischarge heart rate (dichotomised at $70 / \mathrm{min}$ ) and higher versus lower $\beta$-blocker dose (dichotomised at $50 \%$ of the evidencebased target), with the primary composite end point of mortality or cardiovascular rehospitalisation over a median of 2.9 years. All analyses were stratified by the presence of left ventricular systolic dysfunction (LVEF $\leq 40 \%$ ).

Results: After adjustment for covariates, neither $\beta$-blocker dose nor predischarge heart rate was associated with the primary composite end point. However, tachycardia at admission (heart rate $>120$ / $\mathrm{min}$ ) was associated with a reduced risk of the composite outcome in patients with both reduced LVEF (adjusted HR $0.67,95 \% \mathrm{Cl} 0.52$ to $0.88, \mathrm{p}<0.01$ ) and preserved LVEF (adjusted HR $0.79,95 \% \mathrm{Cl} 0.64$ to $0.98, p=0.04$ ).

Conclusions: We found no associations between predischarge heart rate or $\beta$-blocker dosage and clinical outcomes in patients with recent hospitalisations for HF and AF.

\section{INTRODUCTION}

There is extensive evidence that, on average, patients who have heart failure (HF) with reduced left ventricular ejection fraction (LVEF) benefit from $\beta$-blocker therapy. The clinical benefit from $\beta$-blockers is believed to be related in part to their heart rate-lowering

\section{KEY QUESTIONS}

What is already known about this subject?

- Higher $\beta$-blocker dose and lower heart rates are beneficial in patients with systolic heart failure. However, the prognostic impact of these factors in patients with concurrent atrial fibrillation is less clear. Data from meta-analyses and some observational studies suggest that these factors do not have prognostic significance in patients with heart failure and atrial fibrillation.

What does this study add?

- This study demonstrates that neither lower heart rate nor higher $\beta$-blocker dose seem to be important therapeutic targets in patients with heart failure and atrial fibrillation. These findings were consistent in patients with and without a reduction in left ventricular ejection fraction. Additionally, we have identified admission tachycardia as a potential protective factor in these patients, which has not been previously reported.

How might this impact on clinical practice?

- This study argues against the current recommendations of titrating $\beta$-blocker therapy to target doses in patients with recent hospitalisations for heart failure and atrial fibrillation. The association between baseline tachycardia and improved clinical outcomes should be prospectively evaluated, but suggests that more lenient rate control targets could be considered.

effects. ${ }^{1}$ For example, lower baseline heart rates and a larger decrease in heart rate with therapy are associated with better outcomes in outpatient populations. ${ }^{2-4}$ Similarly, in patients admitted for HF, lower discharge heart rate is associated with decreased mortality and readmission rates. ${ }^{5}$ In addition, higher $\beta$-blocker doses lead to greater improvements in $\mathrm{LVEF}^{6-8}$ and may be associated with improved survival, though evidence for the latter is not conclusive. ${ }^{79-11}$ It remains unclear if heart rate-lowering or 
$\beta$-blocker dose is a more important factor for improving clinical outcomes. ${ }^{12}$ Current Canadian, American and European HF guidelines suggest that patients with HF be titrated to target doses from randomised trials. ${ }^{13-15}$

The role of $\beta$-blockers in the $\sim 30 \%$ of patients with $\mathrm{HF}$ who have concomitant atrial fibrillation (AF) is less certain. ${ }^{16}$ Some observational data suggest that patients who are prescribed a $\beta$-blocker do better than those who are not. ${ }^{17}$ However, most available clinical trial and observational evidence does not support survival benefit with $\beta$-blockers in this subgroup. ${ }^{18} 19$ Nevertheless, many patients with $\mathrm{AF}$ and $\mathrm{HF}$ are treated with $\beta$-blockers, which remain recommended first-line agents. ${ }^{14}$ In these patients, the importance of achieving guidelinerecommended dosing and/or heart rate targets is unknown. One large trial showed no advantage with strict versus lenient heart rate targets for patients with $\mathrm{AF}$, including in the subgroup of patients with $\mathrm{HF}^{20}$ Furthermore, whether different $\beta$-blocker dose or heart rate targets are appropriate for patients with $\mathrm{AF}$ and $\mathrm{HF}$ with reduced EF (HFrEF) compared with those with preserved EF (HFpEF) has not been well studied.

To investigate these questions, we performed an analysis of the associations between $\beta$-blocker use and dosing, achieved heart rate, and all-cause mortality in an unselected cohort of patients hospitalised with concurrent diagnoses of $\mathrm{HF}$ and $\mathrm{AF}$.

\section{METHODS}

\section{Study design and data sources}

This is a retrospective analysis using data from an inclusive, prospective observational registry enhanced by detailed chart review and linkage with administrative data sources. Since 2004, the Alberta Provincial Project for Outcome Assessment in Coronary Heart Disease (APPROACH) Heart Alert registry prospectively collects information on patients admitted with cardiovascular diagnoses in the Alberta Health Services Calgary and South zones (population 1.7 million). We used the APPROACH database to identify patients discharged from Calgary area hospitals with concurrent diagnoses of HF and AF between 1 January 2008 and 31 December 2012. Diagnoses are entered into the APPROACH registry at the time of discharge and represent the attending physician's final interpretation of the most responsible diagnosis. We excluded patients whose primary diagnosis was not AF or HF. Since we were interested in the influence of $\beta$-blocker dosage and heart rate at the time of discharge on subsequent events, we excluded patients who died during the index hospitalisation. We only included the first admission during the time period for each patient and also excluded patients who underwent cardiac surgery during the index admission.

The APPROACH data set contains details of patient demographics and comorbidities, cardiovascular interventions and diagnostic tests, as well as cardiovascular medications as previously described. ${ }^{21}$ We accessed the electronic and paper charts for all included patients, to confirm the admitting diagnoses of $\mathrm{HF}$ and $\mathrm{AF}$, and to extract additional data, including admission heart rate, admission and discharge systolic blood pressure, admission and discharge ECG for documentation of AF, measures of LVEF, discharge medications, and discharge laboratory values, including haemoglobin, white cell count, creatinine and sodium. These factors were included based on their importance in predicting survival in patients with $\mathrm{HF}^{22}$ Achieved heart rate was defined as the mean of the last five recorded heart rates. In order to reduce the likelihood of abnormal values being over-represented, we included values to ensure that they were separated by at least 1 hour and spanned at least 24 hours. In order to compare doses of different $\beta$-blockers, we recorded the relative dose, calculated as the total daily dose divided by the target dose used in pivotal trials in HF, expressed as a percentage. ${ }^{9}$ For $\beta$-blockers without an evidence-based target dose in $\mathrm{HF}$, we used the maximum suggested dose as the target. ${ }^{23}$ We defined HFrEF as an LVEF $\leq 40 \%$ based on current guideline definitions. ${ }^{14}$ The recorded LVEF was the last assessment before discharge and was assessed by echocardiogram in $846(89.5 \%)$ patients, MRI in $44(4.7 \%)$ patients, nuclear imaging in $46(4.9 \%)$ patients and not determined in $10(1.1 \%)$ patients. Patients with no determination of LVEF were excluded. Chart review and data extraction were performed using standardised templates, and prior to outcome ascertainment. The Conjoint Health Research Ethics Board of the University of Calgary approved this study, which was conducted in accordance with the Declaration of Helsinki.

\section{Outcomes}

To assess clinical outcomes, we linked the data set with available administrative databases, as previously described. ${ }^{21}$ The primary outcome was the composite of all-cause mortality or hospitalisation with a primary diagnosis of acute coronary syndrome (ACS), AF, HF or stroke. Mortality was ascertained from Alberta Vital Statistics, and hospitalisation outcomes were determined by linkage with the Alberta Health Services Discharge Abstract Database using International Classification of Diseases (ICD)-10 diagnostic codes. The ICD codes used were: ACS (I-20.0, I-20.9, I-21.x, I-22.x, I-24.9, I-25.2), AF (I-48.0, I-48.1, I-48.4, I-48.9) HF (I09.9, I11.0, I13.0, I13.2, I-25.5, I-42.0, I-42.5-9, I-43.x, I-50.x, P29.0) and stroke (G-45.x, G-81.9, H34.0, I-60.x-I69.x) ${ }^{24}$ Secondary outcomes were all-cause mortality, cardiovascular rehospitalisation as defined above, and HF rehospitalisation.

\section{Statistical analysis}

We compared means of continuous variables with the Wilcoxon rank-sum, and proportions with Fisher's exact test. We dichotomised $\beta$-blocker dose at $\geq 25 \mathrm{mg} /$ day carvedilol equivalent, corresponding to $\geq 50 \%$ of the target dose (calculations are outlined in online supplementary table $\mathrm{S} 1$ ), and heart rate at $\geq 70 \mathrm{bpm}$ as previously 
established by Fiuzat et al. ${ }^{10}$ We plotted unadjusted Kaplan-Meier survival curves stratified by $\beta$-blocker and heart rate groups. We then used multivariable-adjusted Cox models to estimate the associations between discharge $\beta$-blocker dose and/or achieved heart rate with the primary composite outcome, as well as the individual secondary outcomes. We tested the proportional hazards assumption with Schoenfeld residuals. All models were constructed separately for HFrEF and HFpEF groups, and were adjusted for age, sex, admission heart rate, LVEF, and all comorbidities, medications, and laboratory values reported in table 1 . We assessed for associations between admission heart rate or change in heart rate during hospitalisation with outcomes. We used a stepwise backwards elimination method to develop a parsimonious final model, we excluded variables with the weakest association until only variables significantly associated $(\mathrm{p}<0.05)$ with the outcome remained. ${ }^{25}$ We forced age, sex, $\beta$-blocker dose and achieved heart rate to remain in the model regardless of significance. We assessed for interactions between variables in the final model and our candidate variables with no significant interactions identified. Two-sided $p$ values $<0.05$ defined statistical significance, and all analyses were performed using Stata V.13 (StataCorp, College Station, Texas, USA).

\section{RESULTS}

We identified 1125 patients in APPROACH who met general inclusion criteria. After chart review, 190 were excluded for the reasons outlined in online supplementary figure S1, leaving a final cohort of 935 patients. Median follow-up was 2.9 years with all surviving patients being followed for at least 1 year. Details of the population characteristics are outlined in table 1 , and a more detailed stratification of characteristics by $\beta$-blocker dose and heart rate groups is shown in online supplementary table S2. The cohort included 409 (43.7\%) patients with HFrEF (mean LVEF 27.2 \pm 8.3 , and 526 (56.3\%) with HFpEF (mean LVEF 55.9 \pm 5.9 ). The achieved heart rate was similar between groups with a median of $72.4 \mathrm{bpm}$ (IQR 64.9-81.5) in the HFrEF group and $72.9 \mathrm{bpm}$ (IQR 64.0-82.7) in the HFpEF group. Of note, only 12 patients were discharged with an average heart rate above

\begin{tabular}{|c|c|c|c|}
\hline Variable & Reduced EF (LVEF $\leq 40 \%) \mathrm{N}=409$ & Preserved EF (LVEF>40\%) N=526 & p Value \\
\hline Male & $305(74.6 \%)$ & $255(48.5 \%)$ & $<0.01$ \\
\hline Age (years) & $68.5 \pm 12.6$ & $75.5 \pm 11.4$ & $<0.01$ \\
\hline Follow-up (years) & $3.0 \pm 1.7$ & $2.8 \pm 1.7$ & 0.09 \\
\hline Myocardial infarction & $126(30.8 \%)$ & $98(18.6 \%)$ & $<0.01$ \\
\hline COPD & $78(19.1 \%)$ & $158(30.0 \%)$ & $<0.01$ \\
\hline Stroke & $38(9.3 \%)$ & $49(9.3 \%)$ & 1.00 \\
\hline Malignancy & $37(9.0 \%)$ & $57(10.8 \%)$ & 0.38 \\
\hline CKD & $66(16.1 \%)$ & $72(13.7 \%)$ & 0.31 \\
\hline Diabetes & $104(25.4 \%)$ & $144(27.4 \%)$ & 0.55 \\
\hline Hypertension & $258(63.1 \%)$ & $368(70.0 \%)$ & 0.03 \\
\hline Dyslipidaemia & $200(48.9 \%)$ & $238(45.2 \%)$ & 0.29 \\
\hline Smoking & $50(12.2 \%)$ & $37(7.0 \%)$ & 0.01 \\
\hline Admission heart rate & 103 (IQR 75-130) & 90 (IQR 69-122) & $<0.01$ \\
\hline Achieved heart rate & 72 (IQR 65-82) & 73 (IQR 64-83) & 0.79 \\
\hline Discharge SBP $(\mathrm{mm} \mathrm{Hg})$ & $113.3 \pm 17.3$ & $121.5 \pm 18.0$ & $<0.01$ \\
\hline Rhythm control & $94(23.0 \%)$ & $95(18.1 \%)$ & 0.07 \\
\hline Amiodarone & $93(22.7 \%)$ & $91(17.3 \%)$ & 0.05 \\
\hline Other antiarrhythmic & $24(5.9 \%)$ & $25(4.8 \%)$ & 0.46 \\
\hline Prescribed $\beta$-blocker & $387(94.6 \%)$ & $418(79.4 \%)$ & $<0.01$ \\
\hline Low dose & 158 (40.8\%) & 165 (39.5\%) & $<0.01$ \\
\hline High dose & $229(59.2 \%)$ & $253(60.5 \%)$ & \\
\hline Metoprolol & $100(25.8 \%)$ & 200 (47.8\%) & $<0.01$ \\
\hline Carvedilol & $212(54.8 \%)$ & $86(20.6 \%)$ & \\
\hline Bisoprolol & $65(16.7 \%)$ & $107(25.6 \%)$ & \\
\hline Other & $10(2.6 \%)$ & $25(5.6 \%)$ & \\
\hline Digoxin & $146(35.7 \%)$ & $126(24.0 \%)$ & $<0.01$ \\
\hline LVEF (\%) & $27.2 \pm 8.3$ & $55.9 \pm 5.9$ & $<0.01$ \\
\hline Haemoglobin (g/L) & $131.1 \pm 20.3$ & $126.0 \pm 19.9$ & $<0.01$ \\
\hline WCC (10^9/L) & $7.1 \pm 2.3$ & $7.4 \pm 3.2$ & 0.71 \\
\hline Sodium (mmol/L) & $138.6 \pm 3.4$ & $139.0 \pm 3.9$ & 0.03 \\
\hline Creatinine (umol/L) & $111.6 \pm 52.9$ & $110.1 \pm 71.7$ & 0.06 \\
\hline
\end{tabular}


110 (6 patients with HFrEF and 6 with HFpEF). The $\beta$-blockers were prescribed at discharge to $815(86.2 \%)$ patients, with $486 \quad(51.4 \%)$ prescribed high-dose $\beta$-blockers. In patients with HFrEF, $\beta$-blockers were prescribed in $387(94.6 \%)$ of patients compared with 418 $(79.4 \%)$ of patients with HFpEF $(\mathrm{p}<0.01)$.

\section{Clinical outcomes}

During follow-up, 331 patients had a total of 538 cardiovascular hospitalisations (390 for $\mathrm{HF}, 70$ for $\mathrm{AF}, 54$ for ACS and 24 for stroke), and 356 patients died. In patients with HFrEF, 140 patients died and 147 patients were rehospitalised on 221 occasions (165 for HF, 21 for AF, 27 for ACS and 8 for stroke). In patients with $\mathrm{HFpEF}$ 216 patients died and 184 patients were hospitalised 317 times (225 for HF, 49 for AF, 27 for ACS and 16 for stroke). Figure 1 shows the unadjusted Kaplan-Meier survival curves for the primary composite outcome and associated HRs, and table 2 presents the final multivariable-adjusted models. There were no significant associations between $\beta$-blocker dosage or predischarge heart rate groups and the primary composite end point of death or cardiovascular rehospitalisation in either the unadjusted or adjusted analyses. Our findings were similar in the reduced and preserved LVEF strata, and were not altered by exclusion of patients who were not prescribed $\beta$-blockers. Table 3 shows the analysis for the
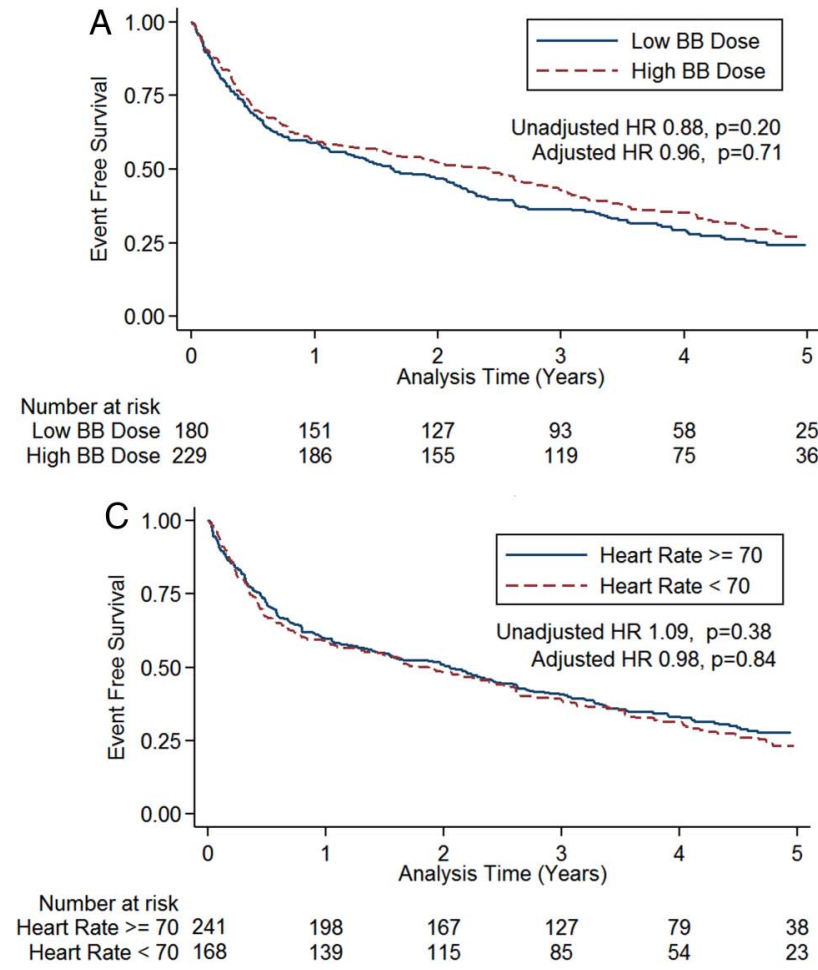

principal secondary end point of all-cause mortality. In this analysis neither $\beta$-blocker dosage group nor predischarge heart rate group was significantly associated with the risk of death, regardless of LVEF stratum. Figure 2 shows Kaplan-Meier survival curves stratified by the presence of tachycardia at admission. Interestingly, admission tachycardia (heart rate $>120 / \mathrm{min}$ ) was associated with a reduction in the composite outcome in both $\mathrm{HFrEF}$ (adjusted HR $0.67,95 \%$ CI 0.52 to $0.88, \mathrm{p}<0.01$ ) and HFpEF (adjusted HR 0.79, 95\% CI 0.64 to $0.98, \mathrm{p}=0.04$ ) groups. In a separate analysis, a greater reduction in heart rate from admission to discharge was also associated with a reduction in the composited outcome in patients with HFrEF (adjusted HR 0.94 per $10 \mathrm{bpm}$ change, 95\% CI 0.90 to 0.97 ) but not in patients with HFpEF.

Several additional clinical factors were associated with the primary composite outcome and/or with mortality, with some variation between the reduced and preserved LVEF groups (see tables 2 and 3). Among these, a discharge prescription for either digoxin (adjusted HR $1.27,95 \%$ CI 1.01 to $1.59 ; \mathrm{p}=0.04$ ) or amiodarone (adjusted HR $1.50,95 \%$ CI 1.17 to $1.91, \mathrm{p}<0.01$ ) was associated with an increase in the primary outcome. There were no statistically significant associations between $\beta$-blocker doses or achieved heart rate groups and the additional secondary outcomes of a readmission for cardiovascular reasons or for $\mathrm{HF}$ (see online

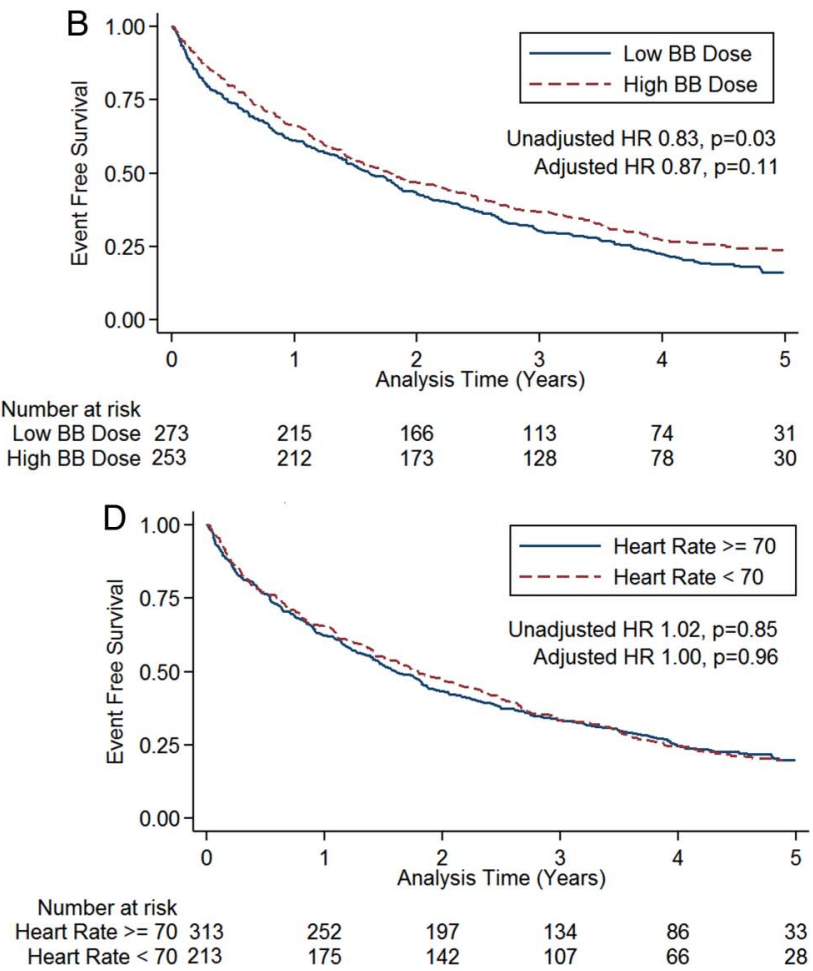

Figure 1 Associations between $\beta$-blocker dosage group, predischarge heart rate group, and the primary composite outcome of death or cardiovascular rehospitalisation. A and B show unadjusted Kaplan-Meier event-free survival estimates for those prescribed $\geq 50 \%$ vs $<50 \%$ of the target dose of $\beta$-blocker, in those with reduced and preserved LVEF, respectively. C and D show unadjusted Kaplan-Meier event-free survival estimates for those whose predischarge heart rate was $\geq 70$ vs $<70 /$ min, in those with reduced and preserved LVEF, respectively. HR: Cox HR. AF, atrial fibrillation; CHF, congestive heart failure; LVEF, left ventricular ejection fraction. 
Table 2 Adjusted Cox models for all-cause mortality or cardiovascular hospitalisation

\begin{tabular}{|c|c|c|c|c|}
\hline \multirow[b]{2}{*}{ Variable } & \multicolumn{2}{|c|}{ Reduced EF (LVEF $\leq 40 \%)$} & \multicolumn{2}{|c|}{ Preserved EF (LVEF>40\%) } \\
\hline & Adjusted HR (95\% Cl) & p Value & Adjusted HR (95\% CI) & p Value \\
\hline Male & $1.23(0.96$ to 1.58$)$ & 0.10 & $0.92(0.77$ to 1.09$)$ & 0.34 \\
\hline Age (per 10 years) & $1.30(1.17$ to 1.44$)$ & $<0.01$ & 1.24 (1.13 to 1.36$)$ & $<0.01$ \\
\hline High $\beta$-blocker dose & $0.96(0.78$ to 1.19$)$ & 0.71 & $0.87(0.73$ to 1.03$)$ & 0.11 \\
\hline Low heart rate (<70 bpm) & 0.98 (0.79 to 1.22$)$ & 0.84 & $1.00(0.84$ to 1.19$)$ & 0.96 \\
\hline Admission tachycardia (>120 bpm) & 0.67 (0.52 to 0.88$)$ & $<0.01$ & 0.79 (0.64 to 0.98$)$ & 0.04 \\
\hline Previous MI & - & - & 1.34 (1.09 to 1.64$)$ & $<0.01$ \\
\hline COPD & $1.32(1.05$ to 1.67$)$ & 0.02 & 1.31 (1.09 to 1.57$)$ & $<0.01$ \\
\hline Malignancy & 1.48 (1.07 to 2.06$)$ & 0.02 & - & - \\
\hline Chronic kidney disease & 一 & - & $1.33(1.04$ to 1.71$)$ & 0.03 \\
\hline Dyslipidaemia & 1.81 (1.45 to 2.27$)$ & $<0.01$ & - & - \\
\hline Liver disease & - & - & $2.21(1.17$ to 4.17$)$ & 0.02 \\
\hline Amiodarone use & $1.50(1.17$ to 1.91$)$ & $<0.01$ & - & - \\
\hline Digoxin use & 1.27 (1.01 to 1.59$)$ & 0.04 & - & - \\
\hline Haemoglobin (per g/L change) & 0.99 (0.98 to 1.00$)$ & $<0.01$ & $0.99(0.99$ to 1.00$)$ & $<0.01$ \\
\hline WCC (per 10^9/L change) & 1.05 (1.01 to 1.09$)$ & 0.03 & - & - \\
\hline Creatinine (per $10 \mu \mathrm{mol} / \mathrm{L}$ change) & $1.04(1.02$ to 1.05$)$ & $<0.01$ & - & - \\
\hline
\end{tabular}

Variable excluded from final model due to non-significance.

Cardiovascular hospitalisation includes heart failure, acute coronary syndrome, atrial fibrillation and stroke. - : Variable excluded from final model due to non-significance. HRs for laboratory data and heart rate represent a change in one unit.

COPD, chronic obstructive pulmonary disease; LVEF, left ventricular ejection fraction; MI, myocardial infarction; WCC, white cell count.

supplementary table S3). The relationship between groups of admission heart rate and the composite outcome is shown in online supplementary figure S2. Patients with admission heart rates $<60 /$ min and $>100 /$ min were less likely to experience the composite outcome compared with patients in the reference heart rate group (60-79/min). Tachycardia at the time of admission was a significant protective predictor in many of these analyses. Patients with tachycardia on admission were younger compared with those without (mean age 69.7 vs 75.3 years, $\mathrm{p}<0.01$ ), but were otherwise similar with respect to baseline characteristics including LVEF $(42.1 \%$ vs $43.8 \%$, $\mathrm{p}=0.13)$. There was a greater decrease in heart rate in this group of patients $(-66 \pm 25$ vs $-8 \pm 19, \mathrm{p}<0.01)$.

\section{DISCUSSION}

In this well-characterised cohort of patients hospitalised with $\mathrm{AF}$ and $\mathrm{HF}$, and with complete, long-term follow-up, we found that while $\beta$-blockers were commonly used, only $60 \%$ of patients were prescribed at least half of the evidence-based target dose. There were no associations between dosing of $\beta$-blockers, or achieved predischarge heart rate, with a composite outcome of all-cause mortality or cardiovascular rehospitalisation, or with the components of this composite. These findings were consistent across the cohorts of patients with and without reduced LVEF. Finally, we found that while predischarge heart rate was not predictive of outcomes, the presence of significant tachycardia at the time of admission was associated with improved prognosis.

\section{Therapeutic targets}

Our study sought to clarify whether $\beta$-blocker dose or achieved heart rate is the more important therapeutic target in patients with HF and AF. While a recent synthesis of subgroups of patients with AF from the pivotal

Table 3 Adjusted cox models for all-cause mortality

\begin{tabular}{|c|c|c|c|c|}
\hline \multirow[b]{2}{*}{ Variable } & \multicolumn{2}{|l|}{ Reduced EF (LVEF $\leq 40 \%)$} & \multicolumn{2}{|c|}{ Preserved EF (LVEF>40\%) } \\
\hline & Adjusted HR (95\% Cl) & p Value & Adjusted HR (95\% Cl) & p Value \\
\hline Male & 1.77 (1.18 to 2.65$)$ & $<0.01$ & $0.96(0.73$ to 1.27$)$ & 0.80 \\
\hline Age (per 10 years) & 1.73 (1.44 to 2.07$)$ & $<0.01$ & 1.71 (1.46 to 2.00$)$ & $<0.01$ \\
\hline High $\beta$-blocker dose & $1.17(0.83$ to 1.766$)$ & 0.37 & $0.82(0.62$ to 1.08$)$ & 0.16 \\
\hline Low heart rate (<70 bpm) & $0.99(0.70$ to 1.41$)$ & 0.96 & $0.90(0.68$ to 1.19$)$ & 0.45 \\
\hline Previous MI & - & - & 1.51 (1.11 to 2.06$)$ & $<0.01$ \\
\hline COPD & $1.47(1.02$ to 2.12$)$ & 0.04 & $1.61(1.21$ to 2.13$)$ & $<0.01$ \\
\hline Dyslipidaemia (per mmol/L) & $1.72(1.21$ to 2.44$)$ & $<0.01$ & - & - \\
\hline Haemoglobin (per g/L) & 0.98 (0.97 to 0.99$)$ & $<0.01$ & 0.99 (0.98 to 0.99$)$ & $<0.01$ \\
\hline WCC (per 10^9/L) & 1.09 (1.03 to 1.16$)$ & $<0.01$ & 1.07 (1.03 to 1.11$)$ & $<0.01$ \\
\hline
\end{tabular}



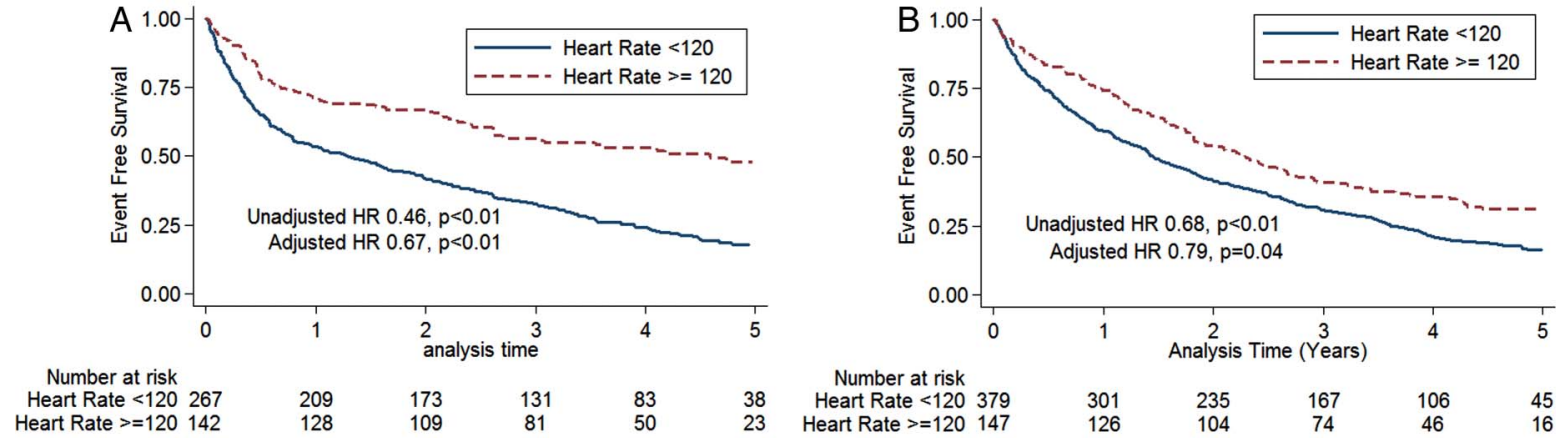

Figure 2 Associations between presence of tachycardia at time of admission (heart rate $\geq 120 / \mathrm{min}$ ) and the primary composite outcome of death or cardiovascular rehospitalisation, in those with reduced (left panel) and preserved (right panel) LVEF. HR: Cox HR. LVEF, left ventricular ejection fraction.

$\beta$-blocker trials in HF has called into question the mortality benefit of $\beta$-blockers in this population, ${ }^{18}$ many patients will require treatment to control ventricular rate and manage symptoms. Our findings of a neutral association between $\beta$-blocker dosage and mortality do not argue against their first-line use in this setting, especially given that non-dihydropiridine calcium channel antagonists are contraindicated in many patients, and that digoxin use has been associated with adverse prognosis in this and other recent studies. ${ }^{26-28}$ This assertion is supported by a recent report from the Swedish HF registry, which reported a similar, significant protective association between $\beta$-blocker use and mortality in patients with HF and reduced LVEF whether in sinus rhythm or $\mathrm{AF}^{29}$ That analysis did not contain information on $\beta$-blocker dosing. However, it did report a graded adverse association between increasing predischarge heart rate and mortality in patients in sinus rhythm, whereas in AF, only a predischarge heart rate $>100 / \mathrm{min}$ was associated with adverse outcomes. Very few patients in our cohort had predischarge heart rates $>100 / \mathrm{min}$, so our results are consistent with this finding. Our results are also congruent with recent reports in outpatients with $\mathrm{HF}$ and $\mathrm{AF}^{30}$ as well as with post hoc analysis of the RACE-II study. ${ }^{20}$ Taken together, these studies suggest that in patients with $\mathrm{HF}$ and $\mathrm{AF}$, there is little rationale to titrate $\beta$-blockers to published dosage targets or to pursue more strict heart rate targets once symptoms are adequately controlled.

It is not clear why the clinical benefits observed by achieving target doses of $\beta$-blockers and target heart rates in those with $\mathrm{HF}$ and sinus rhythm are not seen in the setting of AF, but there are several potential mechanisms. The variability in $\mathrm{R}-\mathrm{R}$ intervals in $\mathrm{AF}$ leads to a reduction in cardiac output and an increase in left atrial pressure independent of effects on heart rate or atrial activity. ${ }^{31}{ }^{32}$ This phenomenon may be particularly prominent in patients with LV dysfunction. ${ }^{33}$ This reduced cardiac output could be further decreased with the acute haemodynamic changes of $\beta$-blocker therapy. ${ }^{34}$ The combination of these effects may lead to adverse haemodynamic changes that prevent patients from achieving the long-term benefits from $\beta$-blocker therapy. This hypothesis could be tested by looking at the effects of $\beta$-blocker therapy in patients with $\mathrm{HF}$ and $\mathrm{AF}$ stratified by cardiac output prior to initiation of therapy. Alternatively, the loss of atrial contraction and the consequently lower stroke volume that occurs when $\mathrm{AF}$ is present may, in the patient with $\mathrm{HF}$, require a higher heart rate to maintain clinical stability. The heart ratelowering effects of $\beta$-blockers in sinus rhythm are primarily through effects on the sinus node, while in AF these changes are through effects on the atrioventricular node. The benefit shown with the use of ivabradine suggests that the beneficial effects of these agents may be specific to effects on the sinus node. ${ }^{35}$ Regardless, further clarification of the mechanisms underlying the differences between patients with $\mathrm{HF}$ in $\mathrm{AF}$ and sinus rhythm, may suggest how to optimise the management of these patients.

\section{Admission tachycardia}

We found that patients admitted with a heart rate $>120 \mathrm{bpm}$ had an improved prognosis, which seems primarily related to a reduction in repeat hospitalisation. This has not previously been reported in this population, to the best of our knowledge. This analysis was not prespecified, and could represent a chance finding. While this group tended to be younger, we also suspect that those with significant tachycardia at admission may be more likely to have $\mathrm{AF}$ as a cause rather than a consequence of HF. This includes the subset of patients with tachycardia-induced cardiomyopathy, a condition known to have a more benign prognosis than other causes of HF once rate and/or rhythm are controlled. ${ }^{36}$ Alternatively, elevated heart rates may provide beneficial haemodynamic compensation during times of stress in these patients. ${ }^{37}$ Finally, patients with tachycardia on admission may have larger changes in heart rate with therapy, a metric known to be important in predicting response to therapy which was also associated with reduced outcomes in patients with $\mathrm{HFrEF}^{4}$ Regardless, this is an interesting finding which should be prospectively validated in future studies. 


\section{Role of $\beta$-blockers in patients with HFpEF and AF}

There is a paucity of data regarding the use of $\beta$-blockers in patients with HFpEF, particularly for patients with concurrent AF. Patient registry and meta-analysis data have not shown an effect of $\beta$-blockers on mortality in patients with HFpEF. ${ }^{18}{ }^{38}$ The Study of the Effects of Nebivolol Intervention on Outcomes and Rehospitalisation in Seniors with Heart Failure (SENIORS) trial showed no significant effect in patients with HFpEF. $^{39}$ However, there was a trend towards a similar effect between patients with HFrEF and HFpEF. ${ }^{39}$ A smaller trial of carvedilol in patients with $\mathrm{HFpEF}$ showed a potential dose-related effect, ${ }^{40}$ while another showed no effect. ${ }^{41}$ In these trials, $\mathrm{AF}$ was present in a minority of patients, further limiting the ability to make any comment about this subgroup. In our real-world population of patients with AF and HFpEF we found no significant effect of $\beta$-blocker dose on clinical outcomes.

\section{Limitations}

Our study has limitations in addition to its observational nature which results in selection bias. Our measure of achieved heart rate was based on nursing assessment and would be better accomplished using 24-hour Holter monitor averages. We used discharge prescription of $\beta$-blockers, with the assumption that patients would continue on these agents at similar doses outside of hospital. This assumption may be valid since previous studies have documented that physicians do not significantly change cardiac regimens after discharge from hospital, ${ }^{42}$ and patients are very likely to fill cardiac medications after discharge ${ }^{43}$ However, heart rates may continue to change after discharge from hospital and assessment of heart rates as assessed at the first outpatient visit may be helpful. We are not able to comment on the effects of very high discharge heart rates due to the low number of patients discharged with heart rates $\geq 100 \mathrm{bpm}$. Finally, we performed several secondary analyses, and all positive results should be interpreted as hypothesis generating.

\section{CONCLUSIONS}

In patients recently discharged from hospital, we found no association between either $\beta$-blocker dose or predischarge heart rate and mortality for patients with $\mathrm{HF}$ and AF. Additionally, we showed that these factors had no impact on subsequent cardiovascular hospitalisations. Patients with tachycardia on admission to hospital may reflect a different population of patients with a more benign outcome.

Competing interests JGH has received speaking or consulting fees from Astrazeneca, Bayer, Novartis, Medtronic, Pfizer, Merck, Servier, CVRx, BMS, and Boehringer-Ingelheim as well as research grants from Astrazeneca, Bayer, Novartis, Medtronic, Pfizer, Merck and Servier. SBW has received consulting fees from Beohringer-Ingelheim and Arca-Biopharma.

Ethics approval University of Calgary Conjoint Health Research Ethics Board.

Provenance and peer review Not commissioned; externally peer reviewed.
Data sharing statement The authors have detailed clinical information on all included patients. These data would be available to authors with original research proposals after appropriate ethics consultation is complete.

Open Access This is an Open Access article distributed in accordance with the Creative Commons Attribution Non Commercial (CC BY-NC 4.0) license, which permits others to distribute, remix, adapt, build upon this work noncommercially, and license their derivative works on different terms, provided the original work is properly cited and the use is non-commercial. See: http:// creativecommons.org/licenses/by-nc/4.0/

\section{REFERENCES}

1. Kjekshus JK. Importance of heart rate in determining beta-blocker efficacy in acute and long-term acute myocardial infarction intervention trials. Am J Cardiol 1986;57:43F-9F.

2. Lechat $\mathrm{P}$, Hulot JS, Escolano $\mathrm{S}$, et al. Heart rate and cardiac rhythm relationships with bisoprolol benefit in chronic heart failure in CIBIS II trial. Circulation 2001;103:1428-33.

3. Vazir A, Claggett B, Jhund P, et al. Prognostic importance of temporal changes in resting heart rate in heart failure patients: an analysis of the CHARM program. Eur Heart J 2015;36:669-75.

4. McAlister FA, Wiebe N, Ezekowitz JA, et al. Meta-analysis: beta-blocker dose, heart rate reduction, and death in patients with heart failure. Ann Intern Med 2009;150:784-94.

5. Habal MV, Liu PP, Austin PC, et al. Association of heart rate at hospital discharge with mortality and hospitalizations in patients with heart failure. Circ Heart Fail 2014;7:12-20.

6. Bristow MR, O'Connell JB, Gilbert EM, et al. Bucindolol Investigators. Dose-response of chronic beta-blocker treatment in heart failure from either idiopathic dilated or ischemic cardiomyopathy. Circulation 1994;89:1632-42.

7. Bristow MR, Gilbert EM, Abraham WT, et al. MOCHA Investigators Carvedilol produces dose-related improvements in left ventricular function and survival in subjects with chronic heart failure. Circulation 1996;94:2807-16.

8. Colucci WS, Kolias TJ, Adams KF, et al. Metoprolol reverses left ventricular remodeling in patients with asymptomatic systolic dysfunction: the REversal of VEntricular remodeling with Toprol-XL (REVERT) trial. Circulation 2007;116:49-56.

9. Bølling R, Scheller NM, Køber L, et al. Comparison of the clinical outcome of different beta-blockers in heart failure patients: a retrospective nationwide cohort study. Eur J Heart Fail 2014;16:678-84.

10. Fiuzat M, Wojdyla D, Pina I, et al. Heart rate or Beta-blocker dose? Association with outcomes in ambulatory heart failure patients with systolic dysfunction: results from the HF-ACTION trial. J Am Coll Cardiol HF 2015;4:109-15.

11. Fiuzat M, Wojdyla D, Kitzman D, et al. Relationship of beta-blocker dose with outcomes in ambulatory heart failure patients with systolic dysfunction results from the HF-ACTION (Heart Failure: a controlled trial investigating outcomes of exercise training) trial. J Am Coll Cardiol 2012;60:208-15.

12. Porapakkham $\mathrm{P}$, Porapakkham $\mathrm{P}, \mathrm{Krum} \mathrm{H}$. Is target dose of beta-blocker more important than achieved heart rate or heart rate change in patients with systolic chronic heart failure? Cardiovasc Ther 2010;28:93-100.

13. McMurray J, Cohen-Solal A, Dietz R, et al. Practical recommendations for the use of ACE inhibitors, beta-blockers, aldosterone antagonists and angiotensin receptor blockers in heart failure: putting guidelines into practice. Eur J Heart Fail 2005;7:710-21.

14. Yancy CW, Jessup M, Bozkurt B, et al. 2013 ACCF/AHA guideline for the management of heart failure. A report of the American College of Cardiology Foundation/American Heart Association Task Force on Practice Guidelines. J Am Coll Cardiol 2013;62:e147-239.

15. McKelvie RS, Moe GW, Ezekowitz JA, et al. The 2012 Canadian cardiovascular society heart failure management guidelines update: focus on acute and chronic heart failure. Can J Cardiol 2013;29:168-81.

16. Mamas MA, Caldwell JC, Chacko S, et al. A meta-analysis of the prognostic significance of atrial fibrillation in chronic heart failure. Eur J Heart Fail 2009;11:676-83.

17. Nielsen PB, Larsen TB, Gorst-Rasmussen A, et al. Beta-blockers in atrial fibrillation patients with or without heart failure: association with mortality in a nationwide cohort study. Circ Heart Fail 2016;9: e002597.

18. Kotecha $D$, Holmes $\mathrm{J}$, Krum $\mathrm{H}$, et al. Efficacy of $\beta$ blockers in patients with heart failure plus atrial fibrillation: an individual-patient data meta-analysis. Lancet 2014;384:2235-43. 
19. Rienstra M, Damman K, Mulder BA, et al. Beta-blockers and outcome in heart failure and atrial fibrillation: a meta-analysis. $J A m$ Coll Cardiol HF 2013;1:21-8.

20. Mulder BA, Van Veldhuisen DJ, Crijns HJ, et al. Lenient vs. strict rate control in patients with atrial fibrillation and heart failure: a post-hoc analysis of the RACE II study. Eur J Heart Fail 2013;15:1311-18.

21. Southern DA, Norris $\mathrm{CM}, \mathrm{Quan} \mathrm{H}$, et al. An administrative data merging solution for dealing with missing data in a clinical registry: adaptation from ICD-9 to ICD-10. BMC Med Res Methodol 2008;8:1.

22. Lee DS, Austin PC, Rouleau JL, et al. Predicting mortality among patients hospitalized for heart failure: derivation and validation of a clinical model. JAMA 2003;290:2581-7.

23. Canadian Pharmacists Association. Compendium of Pharmaceuticals and Specialties. Ottawa: Canadian Pharmacists Association, 2015. http://www.e-therapeutics.ca (accessed online December 2016).

24. Quan $\mathrm{H}$, Sundararajan V, Halfon $\mathrm{P}$, et al. Coding algorithms for defining comorbidities in ICD-9-CM and ICD-10 administrative data. Med Care 2005;43:1130-9.

25. Steyerberg EW, Eijkemans MJ, Harrell FE Jr, et al. Prognostic modelling with logistic regression analysis: a comparison of selection and estimation methods in small data sets. Stat Med 2000;19:1059-79.

26. Turakhia MP, Santangeli P, Winkelmayer WC, et al. Increased mortality associated with digoxin in contemporary patients with atrial fibrillation findings from the TREAT-AF study. J Am Coll Cardiol 2014;64:660-8.

27. Whitbeck MG, Charnigo RJ, Khairy P, et al. Increased mortality among patients taking digoxin-analysis from the AFFIRM study. Eur Heart J 2013;34:1481-8

28. Vamos M, Erath JW, Hohnloser SH. Digoxin-associated mortality: a systematic review and meta-analysis of the literature. Eur Heart $J$ 2015;36:1831-8.

29. Li SJ, Sartipy U, Lund LH, et al. Prognostic significance of resting heart rate and use of beta-blockers in atrial fibrillation and sinus rhythm in patients with heart failure and reduced ejection fraction: findings from the Swedish heart failure registry. Circ Heart Fail 2015;8:871-9.

30. Cullington $\mathrm{D}$, Goode $\mathrm{KM}$, Zhang $\mathrm{J}$, et al. Is heart rate important for patients with heart failure in atrial fibrillation? J Am Coll Cardiol HF 2014;2:213-20.
31. Clark DM, Plumb VJ, Epstein AE, et al. Hemodynamic effects of an irregular sequence of ventricular cycle lengths during atrial fibrillation. J Am Coll Cardiol 1997;30:1039-45.

32. Daoud EG, Weiss R, Bahu M, et al. Effect of an irregular ventricular rhythm on cardiac output. Am J Cardiol 1996;78:1433-6.

33. Iwase M, Aoki T, Maeda M, et al. Relationship between beat to beat interval and left ventricular function in patients with atrial fibrillation. Int $J$ Card Imaging 1988;3:217-26.

34. Felix SB, Stangl V, Kieback A, et al. Acute hemodynamic effects of beta-blockers in patients with severe congestive heart failure: comparison of celiprolol and esmolol. $J$ Cardiovasc Pharmacol 2001;38:666-71.

35. Swedberg K, Komajda M, Böhm M, et al. Ivabradine and outcomes in chronic heart failure (SHIFT): a randomised placebo-controlled study. Lancet 2010;376:875-85.

36. Gopinathannair R, Etheridge SP, Marchlinski FE, et al. Arrhythmia-induced cardiomyopathies mechanisms, recognition, and management. J Am Coll Cardiol 2015;66:1714-28.

37. Rawles JM. What is meant by a "controlled" ventricular rate in atrial fibrillation? Br Heart J 1990;63:157-61.

38. Hernandez AF, Hammill BG, O'Connor CM, et al. Clinical effectiveness of beta-blockers in heart failure. Findings from the OPTIMIZE-HF (organized program to initiate lifesaving treatment in hospitalized patients with heart failure) registry. J Am Coll Cardiol 2009;53:184-92.

39. Flather MD, Shibata MC, Coats AJS, et al. Randomized trial to determine the effect of nebivolol on mortality and cardiovascular hospital admission in elderly patients with heart failure (SENIORS) Eur Heart J 2005;26:215-25.

40. Yamamoto $\mathrm{K}$, Origasa $\mathrm{H}$, Hori M. Effects of carvedilol on heart failure with preserved ejection fraction: the Japanese Diastolic Heart Failure Study (J-DHF). Eur J Heart Fail 2013;15:110-18.

41. Edelmann F, Musial-Bright L, Gelbrich G, et al. Tolerability and feasibility of beta-blocker titration in HFpEF versus HFrEF insights from the CIBIS-ELD trial. JACC Heart Fail 2016;4:140-9.

42. Scherer M, Sobek C, Wetzel D, et al. Changes in heart failure medications in patients hospitalised and discharged. BMC Fam Pract 2006;7:1-6.

43. Jackevicius CA, Li P, Tu JV. Prevalence, predictors, and outcomes of primary. Nonadherence after acute myocardial infarction. Circulation 2008;117:1028-36. 